\title{
SMOOTH EXTENSIONS OF LIPSCHITZIAN REAL FUNCTIONS
}

\section{BIAGIO RICCERI}

(Communicated by Dennis Burke)

Dedicated to Professor F. Guglielmino, with my deepest admiration, on his sixtieth birthday

\begin{abstract}
In this short note we point out that any Lipschitzian real function $f$ defined in a subset $K$ of a Banach space $E$, with $\overline{\operatorname{span}}(K) \neq E$, can be extended to a surjective, open and Lipschitzian real function $g$ on $E$ in such a way that, for every $r \in \mathbf{R}$, the set $g^{-1}(r)$ is arcwise connected. In fact, a more refined result is proved.
\end{abstract}

The aim of this short note is to point out Theorem 1 below. Before giving its statement, we recall that a topological space $Y$ is said to be an absolute extensor for paracompact spaces (see [3]) if, for every paracompact topological space $T$, every closed set $A \subseteq T$ and every continuous function $\psi: A \rightarrow Y$, there exists a continuous function $\varphi: T \rightarrow Y$ such that $\left.\varphi\right|_{A}=\psi$. We also recall that if $U, V$ are two nonempty sets in a normed space $(E,\|\cdot\|)$, their Hausdorff distance, $d_{H}(U, V)$, is defined by putting

$$
d_{H}(U, V)=\max \left\{\sup _{u \in U} \inf _{v \in V}\|u-v\|, \sup _{v \in V} \inf _{u \in U}\|u-v\|\right\} .
$$

Finally, if $E$ is as above, any set $S \subseteq E$ will be regarded as a topological space endowed with the relative norm topology.

THEOREM 1. Let $(E,\|\cdot\|)$ be a real Banach space, $W$ a closed linear subspace of $E$, with $W \neq E, K$ a nonempty subset of $W$ and $f$ a Lipschitzian real function on $K$, with Lipschitz constant $L$. Then, for every $M>2 L$, there exists a real function $g$ on $E$ with the following properties:

(a) $g(x)=f(x)$ for all $x \in K$;

(b) $g$ is Lipschitzian, with Lipschitz constant $(M+2 L) / 2$;

(c) for every $r \in \mathbf{R}$, the set $g^{-1}(r)$ is a nonempty absolute extensor for paracompact spaces;

(d) for every $s, t \in \mathbf{R}$, one has

$$
d_{H}\left(g^{-1}(s), g^{-1}(t)\right) \leq(2 /(M-2 L))|s-t| .
$$

PROOF. By Theorem I of [1], we can extend $f$ to a Lipschitzian real function $\Psi$ on $E$, with Lipschitz constant $L$. Moreover, by a corollary of the Hahn-Banach theorem, there exists a non-null continuous linear functional $\Lambda$ on $E$ such that $\Lambda(x)=0$ for all $x \in W$. Now, for every $x \in E$, put $\Phi(x)=\left(M / 2\|\Lambda\|_{E^{*}}\right) \Lambda(x)$ as

Received by the editors March 5, 1987.

1980 Mathematics Subject Classification (1985 Revision). Primary 54C20, 54C30; Secondary 54C55.

Work supported by M.P.I. 
well as $g(x)=\Phi(x)+\Psi(x)$. The real function $g$, of course, satisfies (a) and (b). Let us show that $g$ satisfies also (c) and (d). To this end, observe that, for every $u, v \in \mathbf{R}$, one has

$$
d_{H}\left(\Phi^{-1}(u), \Phi^{-1}(v)\right)=(2 / M)|u-v| .
$$

Indeed, (1) follows easily from a classical result by G. Ascoli (see Lemma 1.2 of [5]), taking into account that $\|\Phi\|_{E^{*}}=M / 2$. For every $r \in \mathbf{R}$ and $x \in E$, put $F_{r}(x)=\Phi^{-1}(r-\Psi(x))$ as well as $\operatorname{Fix}\left(F_{r}\right)=\left\{y \in E: y \in F_{r}(y)\right\}$. Fix $r \in \mathbf{R}$. Plainly, we have

$$
g^{-1}(r)=\operatorname{Fix}\left(F_{r}\right)
$$

By (1), for every $x, y \in E$, we obtain

$$
d_{H}\left(F_{r}(x), F_{r}(y)\right)=(2 / M)|\Psi(x)-\Psi(y)| \leq(2 L / M)\|x-y\| .
$$

Thus, since $2 L / M<1$, we infer that $x \rightarrow F_{r}(x)$ is a multivalued contraction from $E$ into $E$, with closed convex values. Now, (c) is a direct consequence of (2) and of Theorem 1 of [4]. Finally, fix $s, t \in \mathbf{R}$. Taking into account (1), (2), (3) and Lemma 1 of [2], we then obtain

$$
\begin{aligned}
d_{H}\left(g^{-1}(s), g^{-1}(t)\right) & =d_{H}\left(\operatorname{Fix}\left(F_{s}\right), \operatorname{Fix}\left(F_{t}\right)\right) \\
& \leq \frac{M}{M-2 L} \sup _{x \in E} d_{H}\left(F_{s}(x), F_{t}(x)\right)=\frac{2}{M-2 L}|s-t| .
\end{aligned}
$$

This completes the proof.

REMARK. It is worth noting that, in Theorem 1, (d) implies that the real function $g$ is open. Furthermore, (c) implies that each set $g^{-1}(r)$ is arcwise connected. Lemma 3.4 of $[6]$ then ensures that the set $g^{-1}(A)$ is connected for every interval $A$ of $\mathbf{R}$.

\section{REFERENCES}

1. J. Czipszer and L. Gehér, Extensions of functions satisfying a Lipschitz condition, Acta Math. Acad. Sci. Hungar. 6 (1955), 213-220.

2. T.-C. Lim, On fixed point stability for set-valued contractive mappings with applications to generalized differential equations, J. Math. Anal. Appl. 110 (1985), 436-441.

3. E. Michael, Some extension theorems for continuous functions, Pacific J. Math. 3 (1953), 789806.

4. B. Ricceri, Une propriété topologique de l'ensemble des points fixes d'une contraction multivoque à valeurs convexes, Rend. Accad. Naz. Lincei 81 (1987), 283-286.

5. I. Singer, Best approximation in normed linear spaces by elements of linear subspaces, SpringerVerlag, Berlin and New York, 1970.

6. R. E. Smithson, Multifunctions, Nieuw Arch. Wisk. 20 (1972), 32-53.

Dipartimento di Matematica, Città Universitaria, Viale A. Doria 6, 95125 CATANiA, ItALY 